\title{
KM3NeT - ORCA: Measuring neutrino oscillations and the mass hierarchy in the Mediterranean Sea
}

\author{
Jürgen Brunner*广 \\ Aix-Marseille Université, CNRS/IN2P3, CPPM UMR 7346, 13288, Marseille, France \\ E-mail: brunnerecppm.in2p3.fr
}

The atmospheric flux of neutrinos has traditionally been seen as a background to the detection of an astrophysical neutrino signal. In recent years however, it has been realised that in the few$\mathrm{GeV}$ range, this flux holds a key to resolve a fundamental question of particle physics: that of the neutrino mass hierarchy, i.e. whether the mass eigenstate $v_{3}$ is heavier (normal hierarchy) or lighter (inverted hierarchy) than the $v_{1}$ and $v_{2}$ states.

The influence of the mass hierarchy on neutrino oscillations in matter leaves its imprint on the atmospheric neutrino flux via the characteristic appearance/disappearance patterns of different neutrino types as a function of energy and path through the Earth. ORCA - Oscillations Research with Cosmics in the Abyss - will be a dense configuration of KM3NeT detection units, optimised for studying the interactions of neutrinos in seawater at low energies. To be deployed at the French KM3NeT site, ORCA's multi-PMT optical modules will take advantage of the excellent optical properties of deep seawater to accurately reconstruct both cascade and track events with a few $\mathrm{GeV}$ of energy. This contribution reviews these methods and technology, and compares ORCA's power for not only determining the neutrino mass hierarchy, but placing new constraints on other key parameters such as $\theta_{23}$.

The 34th International Cosmic Ray Conference,

30 July- 6 August, 2015

The Hague, The Netherlands

* Speaker.

${ }^{\dagger}$ on behalf of the KM3NeT Collaboration 


\section{Introduction}

Past experiments, using solar, atmospheric and man-made neutrinos, have successfully measured three mixing angles and two absolute mass square differences between the neutrino mass eigenstates. Global fits of all available input from experiments provide a coherent picture within the three-flavour oscillation framework [1]. However, the picture is incomplete. Among the yet unknown features of the neutrino sector figures the ordering of the neutrino masses. Conventionally, $v_{1}$ denotes the mass eigenstate which couples most strongly to the electron and correspondingly $v_{3}$ has the weakest coupling to this state. From experiments with solar neutrinos it is known that $m_{v_{2}}>m_{v_{1}}$ due to matter effects which modify the oscillation probabilities of the neutrinos on their path through the Sun. Experiments with atmospheric neutrinos or with man-made neutrino sources on Earth were so far unable to detect Earth matter effects or to reveal sub-leading effects of a full three-flavour treatment. It is therefore currently unknown whether $m_{v_{3}}>m_{v_{2}}$ (normal mass hierarchy - NH) or $m_{v_{1}}>m_{v_{3}}$ (inverted mass hierarchy - IH). Unlike other unknowns in the neutrino sector, such as the value of the CP-phase or the Majorana/Dirac nature of neutrinos, the neutrino mass hierarchy $(\mathrm{NMH})$ is considered to be in reach for experiments before the end of this decade. It has been proposed to determine the NMH by measuring matter effects of atmospheric neutrinos in the Earth in Mton water Cherenkov detectors [2]. Matter effects modify the oscillation pattern in the opposite way for neutrinos and antineutrinos as well as for $\mathrm{NH}$ and IH. The effect is expected to be easily detectable at the Earth mantle resonance energy of about $6 \mathrm{GeV}$. While the envisaged water Cherenkov detectors cannot distinguish between neutrinos and antineutrinos due to the absence of a magnetic field, the atmospheric neutrino fluxes are slightly larger than antineutrino fluxes and the neutrino cross sections are about a factor two higher than those for antineutrinos. This ensures a non-vanishing Earth matter differential effect between the two NMH hypotheses.

\section{The ORCA Detector}

The successful deployment and operation of the ANTARES neutrino telescope [3] has demonstrated the feasibility of performing neutrino studies with large volume detectors in the deep sea. The KM3NeT [4] technology builds upon this experience and improves the cost effectiveness of the design by about a factor four. The 31 photo-sensors and the readout electronics are hosted within pressure resistant glass spheres, so called Digital Optical Modules (DOMs) [5]. More details on the DOMs can be found also in these proceedings in the contribution by Ronald Bruijn. The DOMs are distributed in three dimensional grid along flexible strings, one end of which is fixed to the sea floor and the other end is held close to vertical by a buoy. A building block of the KM3NeT detector consists of 115 such vertical strings, each equipped with 18 DOMs. The ORCA detector will consist of one such building block. The sensitive energy range of the detector is mostly determined by the photo-sensor density. The same detector units as used for the high energy neutrino telescope, optimised for $\mathrm{TeV} / \mathrm{PeV}$ energies, can therefore also be used for ORCA with a different vertical spacing of the DOMs along the strings and a smaller inter-string distance. The latter is fixed to values greater than about $20 \mathrm{~m}$ by deployment constraints. The vertical spacing is currently being optimised in the range $6 \mathrm{~m}$ to $18 \mathrm{~m}$. Correspondingly, the instrumented water mass ranges from about 3.75 Mtons to 11.25 Mtons. If not otherwise mentioned, the results be- 
low are obtained with an inter-string distance of $20 \mathrm{~m}$ and a vertical DOM spacing of $6 \mathrm{~m}$. The design provides nanosecond precision on the arrival time and the time-over-threshold of all single photons. Calibration units provide tools to achieve the necessary precision in the position measurement (few centimetres) and orientation measurement (few degrees) of the photo-sensors based on experience with ANTARES. The string concept is modular by design thereby facilitating a phased and distributed implementation.

The ORCA detector will be located $40 \mathrm{~km}$ offshore from Toulon, France, about $10 \mathrm{~km}$ East of the existing ANTARES telescope, at a depth of $2475 \mathrm{~m}$. The first main electro-optical cable was deployed successfully in December 2014 and the first junction box needed to connect strings has been deployed and connected in summer 2015. Funds have been obtained to build, deploy and operate the first 6 strings which is foreseen until the end of 2016. Once more funds become available, the full ORCA array will be constructed and become operational within 3 years.

\section{Simulation Tools and Reconstruction Methods}

Neutrino and antineutrino induced interactions in the energy range from 1 to $100 \mathrm{GeV}$ have been generated with a software package based on the GENIE [6] neutrino event generator. The events are weighted to reproduce the conventional atmospheric neutrino flux following the Bartol atmospheric neutrino flux model [7]. The background due to down-going atmospheric muons is generated with the MUPAGE [8] program. MUPAGE provides a parameterised description of the underwater flux of atmospheric muons including also multi-muon events. All particles in the vicinity of the equipped detector volume are propagated with a GEANT based software package. It generates Cherenkov light from primary and secondary particles and simulates hits taking into account the light absorption and scattering in water as well as the DOM and PMT characteristics. The optical background arising from the decay of radioactive ${ }^{40} \mathrm{~K}$ in seawater is taken into account.

Two methods are being developed to reconstruct neutrino events with apparently different signatures: a track and a cascade reconstruction. The track reconstruction is designed to identify muon tracks created by charged current (CC) interactions of $v_{\mu}$, whereas the cascade reconstruction focuses on $v_{e}$-CC interactions. However, most other interaction channels yield as well a cascade signature. A neutrino interaction is fully described by eight parameters: its vertex position (3), time (1), direction (2), energy (1) and inelasticity (1) - defined as the fraction of the neutrino energy which is transferred to the hadronic system. To determine all parameters, both methods use a multi-step approach: In the two initial steps the lepton direction and the vertex position are obtained in several iterations between these two steps. To obtain the neutrino energy, the total light yield is exploited in conjunction with the already determined vertex position and lepton direction. The inelasticity is estimated from the ratio of the direct Cherenkov light (close to the theoretical Cherenkov angle with respect to the fitted lepton direction) compared to off-angle light, which has a higher probability to be emitted by hadrons. The effect of the inelasticity on the angular distribution of the emitted photons is illustrated in Figure 1 (left). Neutrino interactions are only kept for subsequent analysis steps, if their reconstructed vertex is within a fiducial volume and the majority of the produced light is seen by the detector. The measurement of the NMH is normally done by examining the event yield as function of neutrino energy and zenith angle [2]. However, it has been demonstrated, that the sensitivity can be improved by additionally using the inelasticity [9]. More 

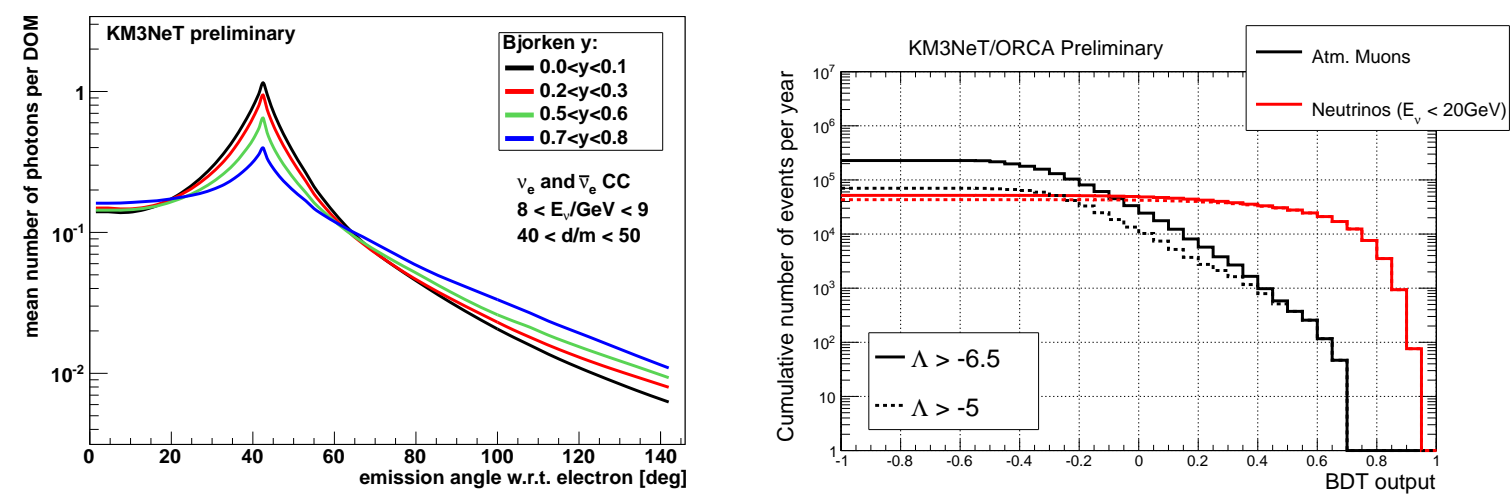

Figure 1: Left: Angular distribution of emitted photons with respect to the true electron direction for $v_{e}$-CC interactions for different inelasticities, Right: Output of the BDT algorithm to distinguish misreconstructed atmospheric muons from up-going $v_{\mu}$-CC events after applying a containment criteria. Dashed and full lines are for different choices of the track-fit quality cut.

details on the two reconstruction methods (cascades and tracks) can be found in these proceedings in the contributions by Jannik Hofestädt and Salvatore Galata.

For the NMH determination a pure sample of well-measured up-going atmospheric neutrinos is needed. Misreconstructed down-going atmospheric muons can potentially pollute this sample. After applying basic cuts on the reconstructed zenith angle (up-going) and the vertex position (containment criteria) a boosted decision tree (BDT) is applied to ensure the separation of the neutrinos from atmospheric muons. The BDT output variable is shown on Figure 1 (right). A contamination of a few percent of atmospheric muons can be reached, keeping a high efficiency for the neutrino sample. This is subject to further optimisation.

The above mentioned algorithms have been tuned on samples of $v_{\mu}$-CC and $v_{e}$-CC events. However, in real data both are mixed, moreover other types of events occur as well, such as neutral current (NC) events. Therefore it is important to distinguish the two main event topologies, to allow their separate reconstruction and analysis. To this end a machine learning algorithm is used, a random decision forest (RDF [10]), that is trained on Monte Carlo events to distinguish between the two classes, track-like and cascade-like events. A RDF consists of many decision trees that individually categorise an event into different classes and whose decisions are combined into a majority vote with a configurable threshold. The algorithm relies on several discriminating observables that combine information extracted from the event. The results of the above described reconstruction algorithms, in particular their fit qualities, are key ingredients to the correct classification of a cascade-like or track-like event.

\section{Performance}

Deep sea water is characterised by an effective scattering length of more than $200 \mathrm{~m}$ in the relevant wavelength range for ORCA. Therefore the majority of the detected photons from neutrino interactions inside the equipped volume are unscattered Cherenkov photons. This feature is exploited in the reconstruction procedures and yields a zenith angle resolution better than $10^{\circ}$ for 

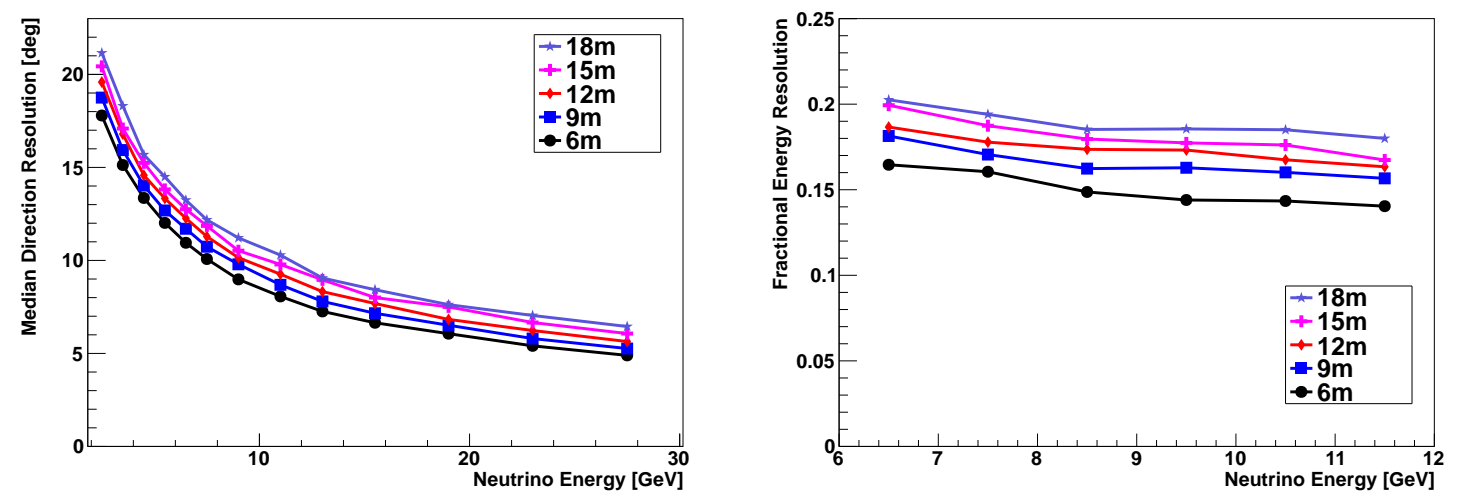

Figure 2: Dependence of resolution functions of $v_{e}$-CC events on vertical spacings of the DOMs, Left: Median zenith angle distribution, Right: Fractional neutrino energy resolution.

neutrino energies above $10 \mathrm{GeV}$. At lower energies the resolution is dominated by the kinematic angle between neutrino and charged lepton. The angular resolution for different vertical spacings of the DOMs along the detector strings is shown in Figure 2 (left) for $v_{e}$-CC events. It depends only weakly on the vertical spacing in the considered range of 6-18 m. For $v_{\mu}$-CC even slightly better results are obtained.

The neutrino energy is determined by exploiting the total light yield of the events. Therefore the energy resolution for the two signal samples $\left(v_{\mu}-\mathrm{CC}\right.$ and $\left.v_{e}-\mathrm{CC}\right)$ is better than what could be expected by merely reconstructing the charged lepton emerging from the interaction point. A close-to Gaussian resolution function is obtained for the selected event samples in the relevant energy range from $5-12 \mathrm{GeV}$ with a width of $15-20 \%$ for $v_{e}$-CC (see Figure 2 (right) ) and around $25 \%$ for $v_{\mu}$-CC. The dependence of the resolution on the vertical spacing of the DOMs is moderate, as illustrated as well in Figure 2 (right).

The result of the event classification RDF is shown in Figure 3 (left). The probability to classify as track-like is shown for all reaction channels. For relevant neutrino energies of $10 \mathrm{GeV}$, about $10 \%$ of the events in the cascade signal channels $v_{e}, \bar{v}_{e}$-CC are mis-classified as tracks, whereas $67 \%$ and $88 \%$ of $v_{\mu}$-CC and $\bar{v}_{\mu}$-CC are correctly classified as such, respectively.

On Figure 3 (right) the effective mass for the selected event samples is shown as function of the neutrino energy for different vertical spacings of the DOMs. The asymptotic values at large neutrino energies illustrate the increase in instrumented volume for a constant number of DOMs while increasing the vertical spacing between them. However, this increase is accompanied by a shift in the effective energy threshold towards higher energies. The optimisation of the vertical spacing in terms of the sensitivity in determining the $\mathrm{NMH}$ is currently under study.

\section{Systematic Effects}

The determination of the NMH is a high precision measurement. Each year more than ten thousand neutrino interactions will be detected by ORCA. The distinction of the two NMH hypotheses $\mathrm{NH} / \mathrm{IH}$ is based on few percent differences in the event count for certain regions in the energy-angle-inelasticity space. To succeed, a very good control of systematic uncertainties is 

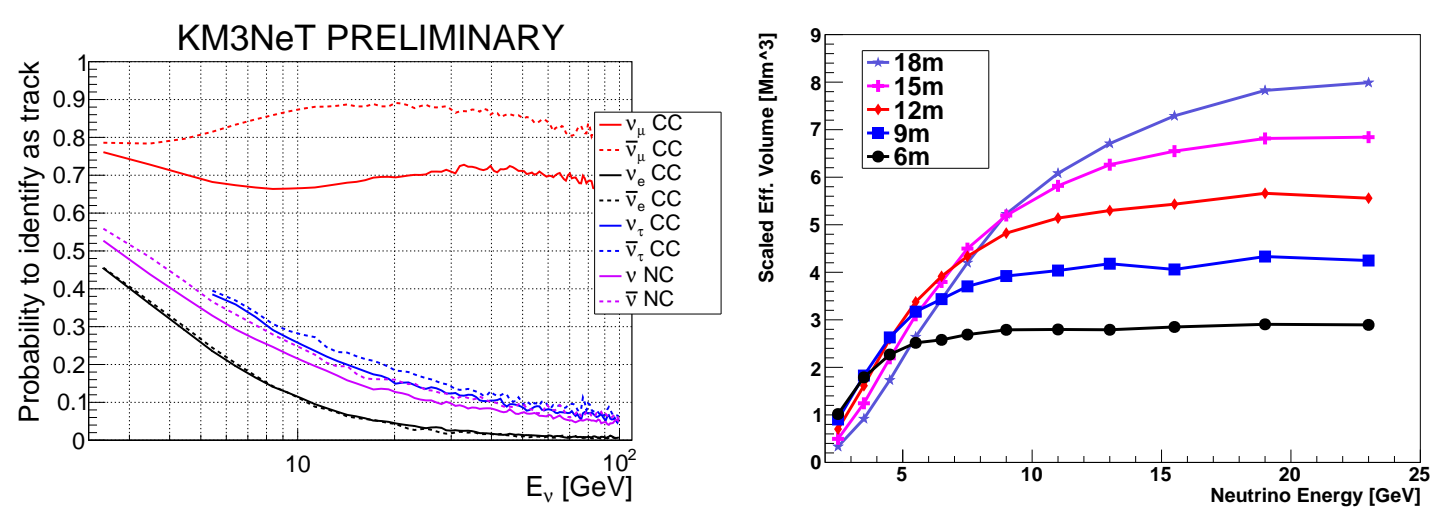

Figure 3: Left: Fraction of events classified as tracks for the different reaction channels, Right: Effective mass as function of neutrino energy for different vertical spacings of the DOMs.

needed. A first class of such uncertainties is related to the detector itself. The absolute efficiencies of the photo-sensors must be permanently monitored over the lifetime of the experiment. It has been shown by ANTARES, that this can be done, using coincidence signals from ${ }^{40} K$ decays, which can be seen as a natural occurring very stable calibration source. As only decays in the vicinity of a DOM contribute to these coincidences, the ${ }^{40} \mathrm{~K}$ calibration can be done independently from assumptions on water properties. These instead have to be monitored as well by different means. LED and laser beacons are used to measure the absorption length. Light scattering is expected to play only a marginal role due to the limited size of ORCA compared to high-energy neutrino telescopes.

A second class of uncertainties is not detector related. Uncertainties on neutrinos fluxes, their cross sections as well as on the modeling of the hadronisation fall in this category. They are shared by all experiments with similar goals and methods. Most of the resulting uncertainties can be parametrised in the form of nuisance parameters and incorporated into the global fit. The leading effect of all the mentioned uncertainties is the modification of the total event count. This is absorbed into a free, unconstrained normalisation. Other effects are taken into account as energy scale factors, an energy slope correction $E_{v}^{\mathcal{\varepsilon}}$ as well as modifiable ratios of different event classes $(v / \bar{v}$, track/cascade, NC/CC). Preliminary studies indicate that none of these effects compromise substantially the ability of ORCA to determine the NMH.

Finally the uncertainties of some of the neutrino oscillation parameters themselves have an impact on the measurement. The mixing angle $\theta_{12}$ and $m_{v_{2}}^{2}-m_{v_{1}}^{2}$, which control solar neutrino oscillations, do not influence the NMH measurement, so they are fixed to their world best values. The angle $\theta_{13}$, determined in reactor experiments, is allowed to vary with a Gaussian prior within its current uncertainty. ORCA cannot improve the precision of $\theta_{13}$ but a larger value increases the sensitivity of the NMH measurement. The mass splitting $\left|\Delta m_{32}^{2}\right|$ (or equivalently $\left|\Delta m_{31}^{2}\right|$ ) as well as $\theta_{23}$ can both be measured together with the NMH. Therefore both values are fitted together with the NMH without constraining priors. It is found that a certain level of degeneracy exists between the NMH hypotheses and the octant choice $\theta_{23}<45^{\circ}$ versus $\theta_{23}>45^{\circ}$. The reachable precision for both parameters is shown in Figure 4. For $\theta_{23}$ a true value of $42^{\circ}$ is assumed. ORCA could 

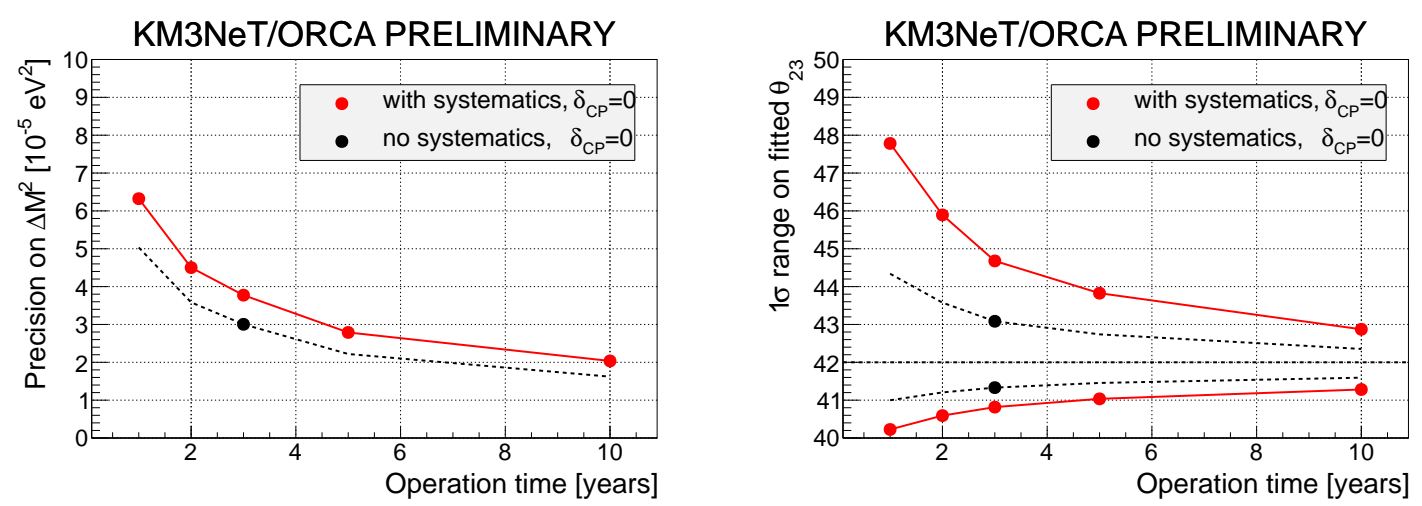

Figure 4: Measurement precision (68\% C.L.) as function of running time of ORCA. The impact of systematic uncertainties is illustrated by comparing dashed and solid lines, Left: $\Delta m_{32}^{2}$ Right: $\theta_{23}$.

substantially improve the precision for both parameters.

\section{Mass Hierarchy Sensitivity}

The NMH sensitivity is determined by examining event numbers from pseudo-experiments in 20 bins in $\log \left(E_{V}\right)$ and 20 bins in $\cos \vartheta$, the measured zenith angle. The inelasticity is currently not used. Track-like and cascade-like samples are treated separately. A likelihood ratio is calculated by testing both hypotheses $(\mathrm{NH} / \mathrm{IH})$ on the event distributions. At the same time, the oscillation parameters and the above discussed nuisance parameters are fitted as well. This is repeated for a large number of pseudo-experiments for different sets of input parameters. This allows to determine the separation of the two hypotheses and therefore its reachable sensitivity. Figure 5 shows the evolution of the sensitivity with time. Both cascade and track channels are used. The cascade channel is crucial for the overall distinction power between the two hypotheses. However, the track-channel is indispensable in constraining the oscillation parameters.

\section{Conclusion}

The ORCA experiment aims at measuring matter-induced modifications of neutrino flavor oscillation probabilities by the detection of atmospheric muon and electron neutrinos that have traversed the Earth interior. Differences in the zenith angle and energy dependent count rates open the possibility to determine the neutrino mass hierarchy. Detailed detector simulations show that the long scattering length of light in water allows attaining promising direction and energy resolutions for both channels and indicate sensitivity to the reaction inelasticity which will be exploited in further studies. Employing detailed detector response functions for both signal and background, the median sensitivity is estimated to about $3 \sigma$ in 3 years assuming the current best fit value for the mixing angle $\theta_{23}$ in the first octant.

In a first construction phase, the deep-sea neutrino telescope technology developed in the $\mathrm{KM} 3 \mathrm{NeT}$ project will be employed to construct and operate a densely instrumented 6 string detector off the coast of Toulon, France. It will serve to demonstrate the feasibility of the measurement and to validate and optimise the detector design. 


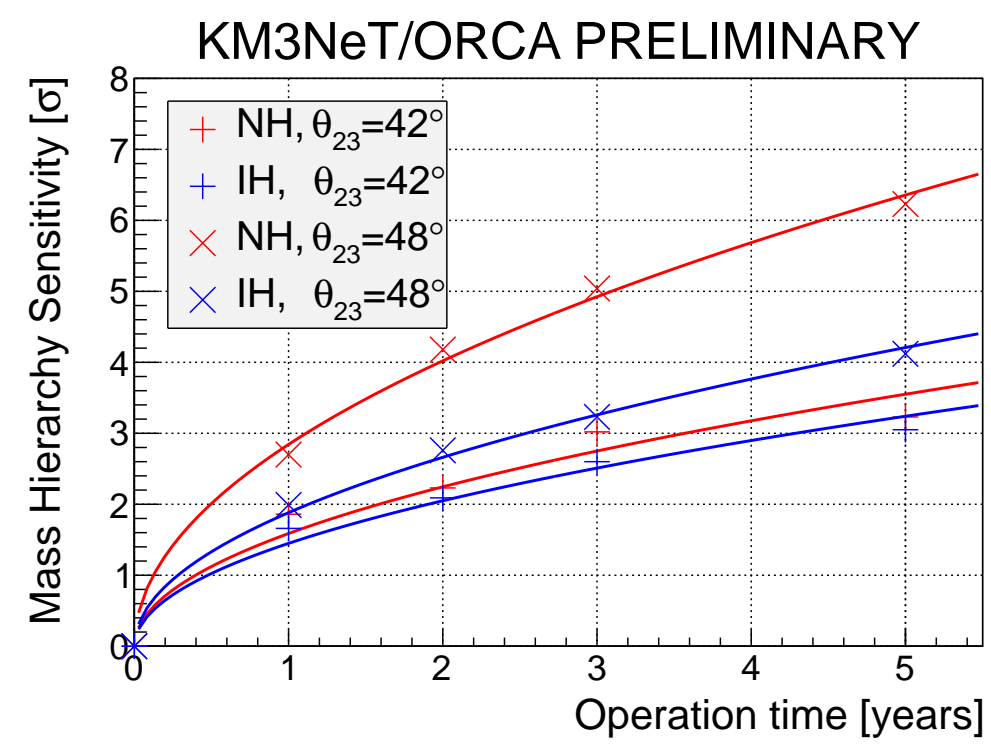

Figure 5: NMH sensitivity as function of data taking time with ORCA for both NMH hypotheses and two values of $\theta_{23}$. With the exception of the CP-phase all considered systematic uncertainties are taken into account.

\section{References}

[1] G.L. Fogli et al., Global analysis of neutrino masses, mixings and phases: entering the era of leptonic CP violation searches, Phys. Rev. D 86 (2012) 013012.

[2] E. Kh. Akhmedov, S. Razzaque and A. Yu. Smirnov, Mass hierarchy, 2-3 mixing and CP-phase with Huge Atmospheric Neutrino Detectors, JHEP 02 (2013) 082.

[3] M. Ageron et al., ANTARES: the first undersea neutrino telescope, NIM A 656 (2011) 11.

[4] A. Margiotta et al., The KM3NeT deep-sea neutrino telescope, NIM A 766 (2014) 83.

[5] S. Adrian-Martinez et al., Deep sea tests of a prototype of the KM3NeT digital optical module, EPJ C 74 (2014) 3056.

[6] C. Andreopoulos et al., The GENIE Neutrino Monte Carlo Generator, NIM A 614 (2010) 87.

[7] G. D. Barr et al., Three-dimensional calculation of atmospheric neutrinos, Phys. Rev. D 70 (2004) 023006.

[8] G. Carminati et al., An update of the generator of atmospheric muons from parametric formulas (MUPAGE), Comp. Phys. Comm. 181 (2010) 835.

[9] M Ribordy and A. Yu. Smirnov, Improving the neutrino mass hierarchy identification with inelasticity measurement in PINGU and ORCA, Phys. Rev. D 87 (2013) 113007.

[10] L. Breiman, Random Forests, Machine Learning 45 (2001) 5. 